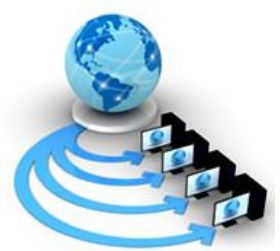

Volume 11, No. 3, May-June 2020

ISSN No. 0976-5697

International Journal of Advanced Research in Computer Science

RESEARCH PAPER

Available Online at www.ijarcs.info

\title{
A SOFTWARE TOOL FOR RECOVERING LOST MOBILE PHONES USING REAL-TIME TRACKING
}

\author{
Iwara I. Arikpo \\ Department of Computer Science \\ University of Calabar \\ Calabar, Nigeria
}

\author{
Gabriel I. Osuobiem \\ Department of Computer Science \\ University of Calabar \\ Calabar, Nigeria
}

\begin{abstract}
The rapid increase in the use of smartphones and other mobile devices in developing countries like Nigeria, comes with the huge challenge of rampant phone theft and difficult process of recovery. This study was aimed at improving the recovery process for lost phones by the development of a mobile application for locating and retrieving lost mobile phones in real-time. The system design methodology was based on object-oriented analysis and design using the unified modelling language. The system was implemented using Android SDK Tools Version 23.0.5 in combination with Google Map service and an underlying Firebase real-time database. The application was tested with Android 5.0 (Lollipop). The software was successfully used to track mobile devices in real-time with other retrieval aids like lock, ring and wipe (if the owner wants to) applied during the recovery process. The authors recommend this application as a simplified solution to the problem of mobile phone theft/misplacement.
\end{abstract}

Keywords: mobile phone, tracking, android, recovery, L-Code

\section{INTRODUCTION}

In the past few years, computers have been tremendously miniaturized. Advances in information and communication technology have led to the development of various handheld and pocket-sized telecommunication devices, whose importance in the current global information technology economy cannot be overemphasized. The mobile phone, being the most common form of such devices possess extensive computing capabilities, including high-speed access to internet services regardless of its small size [1].

Today, mobile phones have become almost indispensable because of the ease at which they can be carried around, and readily integrated into a user's routine. Connecting with work, family and friends has been made easy with the use mobile phones. In 2016, an estimated $62.9 \%$ of the population worldwide already owned a mobile phone. The mobile phone penetration is forecasted to continue to grow, rounding up to $67 \%$ by 2019 , [2]. In Nigeria, for example, smartphone users have been forecast to grow to over 140 million $(60 \%)$ by 2025 [7].

This paper titled improving/simplifying the recovery process for lost/stolen phones is an Android application that will serve as a tool for reuniting users with their lost phones. With the use of Google Maps and an underlying Firebase real-time database, the application will provide live and accurate location data of the lost device. Finding a phone is one thing and retrieving it is another, in a scenario where a user tracks down the lost phone using the application, the user can also perform administrative operations such as data wipe, device lock, and device ring remotely. With the device ring feature, the user will be able to ring the lost phone even if the phone is on silent mode.

\section{LITERATURE REVIEW}

Attempts have been made by several researchers towards the development of an efficient mobile phone tracking system. [3], developed an Anti-Theft Application for Lost or Misplaced Android Phones. The application was designed to enable users track their lost or misplaced devices. This was made possible with the use of GPS tracking, video recording, image capturing, SIM card change notification, SMS, MMS, and email messaging. The video recording and image capturing feature of the application is an added advantage; these features create a possibility of identifying the possible thief, if the phone was stolen. Limitations of the application include, unavailability of a feature that plots the GPS coordinates of the device and presents it on a map, as well as the inability of the application to provide real-time data.

Google LLC released an "Android Device Manager" application that allows users to find their smartphones if stolen or misplaced. It was attached to the Google account of every user, so as to make it available for anybody that owns a Google account [4]. The application is available on the web and also on Android. Users can lock, wipe, and ring their phone from another device (either smartphone or PC) remotely. A real-time map is also available with a marker on the current location of the phone when online, or the last recorded location before going offline [5].

This application turns out to be redundant if the user is logged out of his/her Google account, it loses control over the lost device. For this reason, the developed application of this will not allow a user to be logged out, and may prevent uninstallation of the application.

[6] also designed an application called Mobile Tracker App, that could report the current location, and other vital information about a lost mobile phone. The application gives a user the ability to perform several operations on the 
misplaced device. This is done through text messages sent from another device with an alternatively registered phone number. Text messages cannot be sent from any phone, the application has two flavours, one for the misplaced device, and the other for the control device. To the application, "@tracklocation" means fetch current location and send (in longitude and latitude), “@takepicture” means take a picture and send to registered email, "@trackcontacts" means send contact list, etc. Whenever the SIM card of the lost device is changed, Mobile Tracker App sends a text message containing information about the newly inserted SIM card to the control device.

The strength of this application is that it is operable and functional without the need for any internet connection. Since the lost device can be controlled exclusively via text messaging.

The main drawback of this tracking solution is its inability to ensure recovery. It will be very difficult to recover a lost phone without the availability of a map, because users may not know what to do with the GPS coordinates sent to them. Another limitation is that, the application only activates the rear (back) camera of the device, therefore, making it hard for it to capture the face of any possible thief.

\section{Methodology}

The design of the application developed in this study is based on the Object-Oriented Analysis and Design methodology (OOAD) using the Unified Modelling Language (UML). The system design components are shown in Figures 1 - 4.

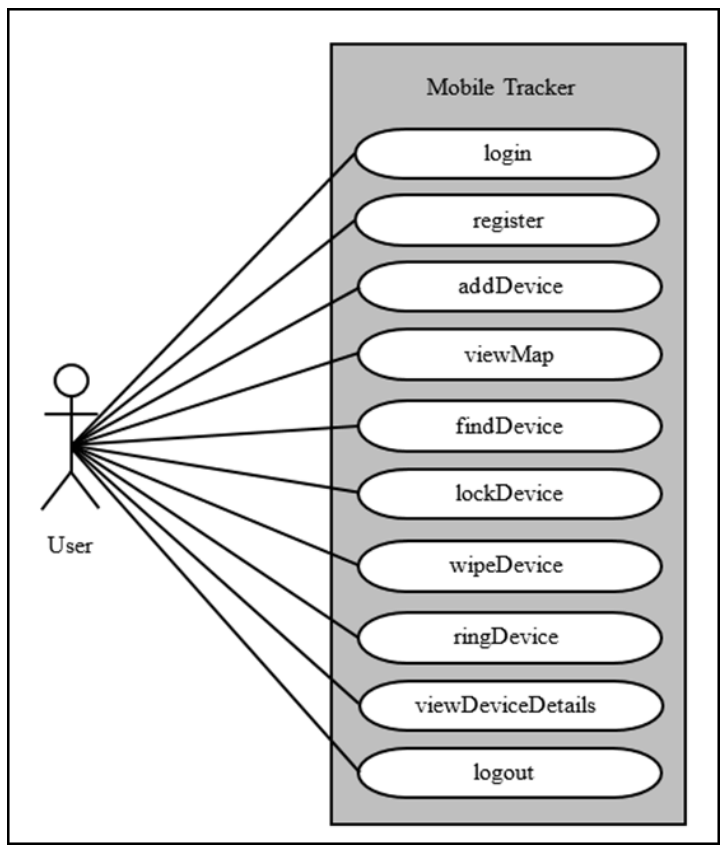

Figure 1: Use Case Diagram

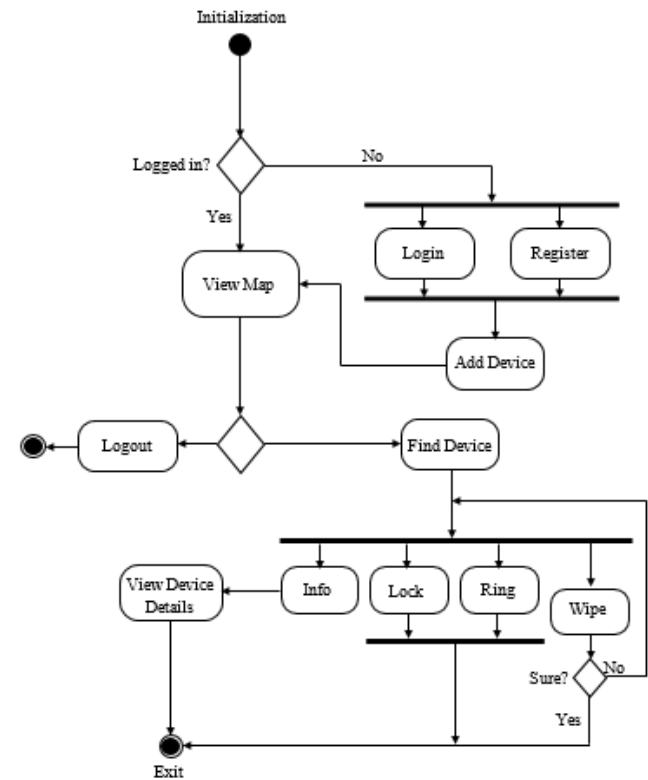

Figure 2: Activity Diagram

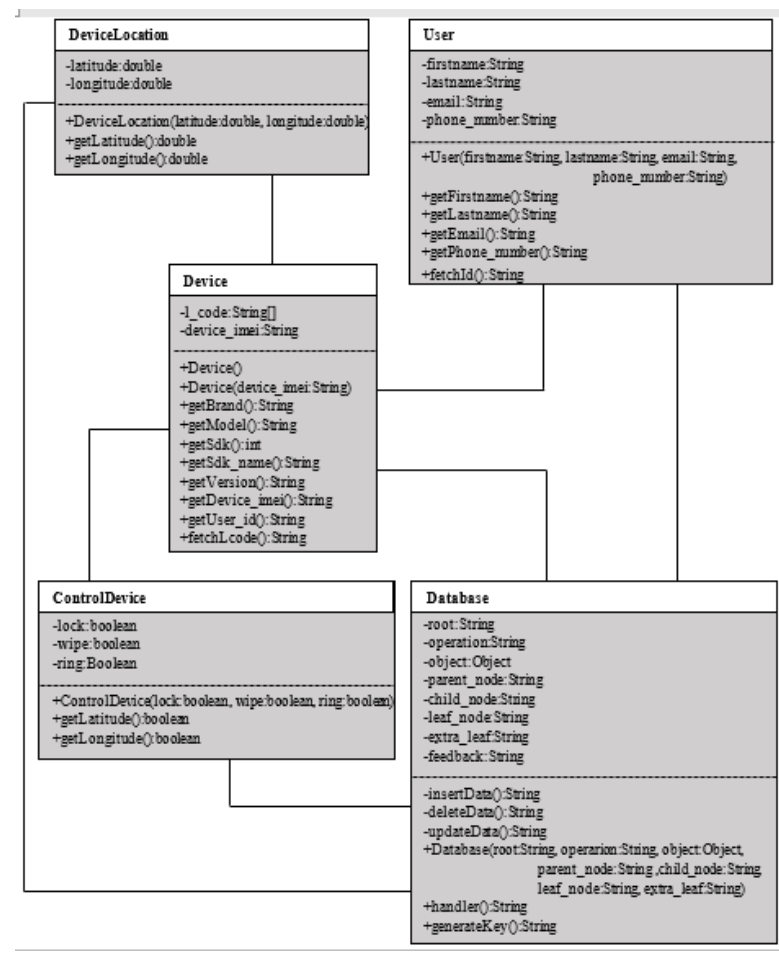

Figure 3: Class Diagram 


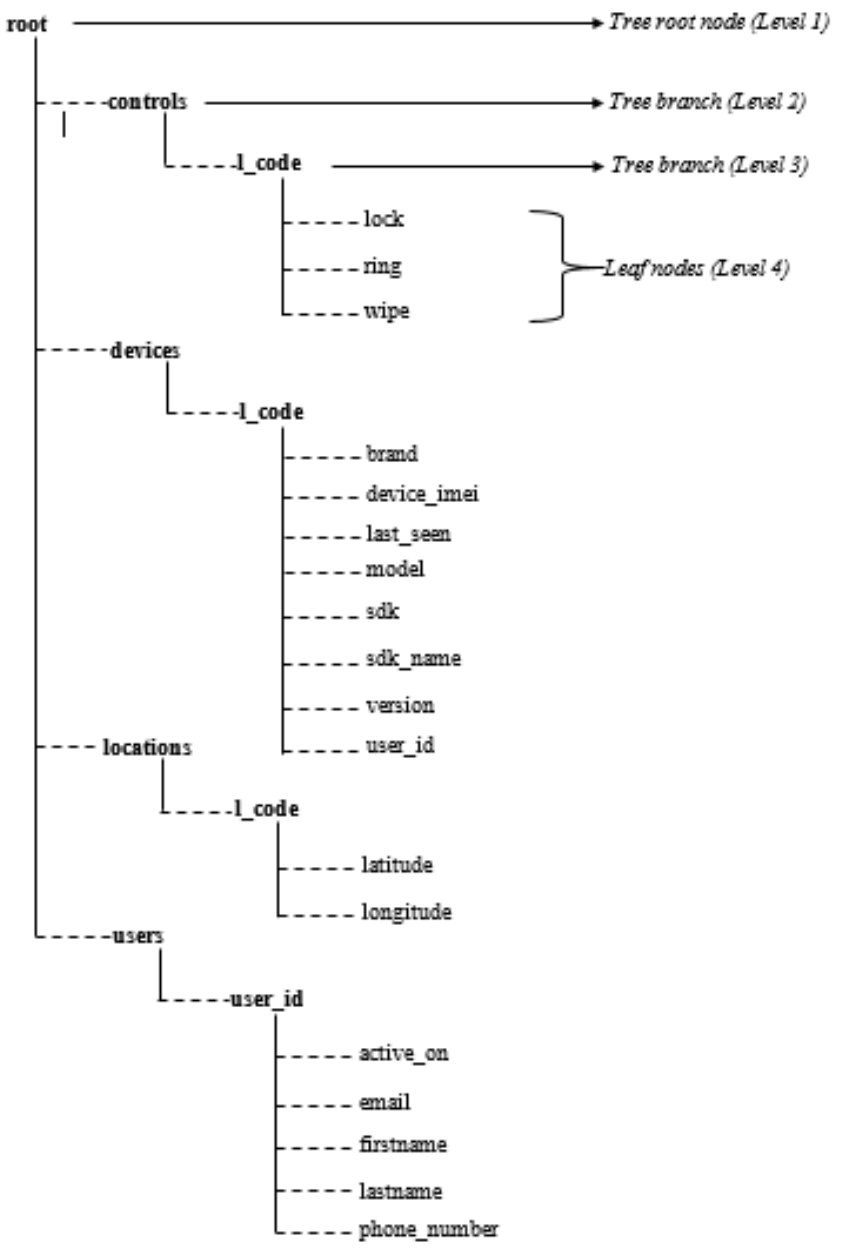

Figure 4: Database Structure

The application was developed with the official IDE for Android application development Android Studio SDK, which comes with all the necessary tools for developing Android applications. The logic of the application program is written in Java, while the layout and interface design are written in XML (eXtensible Markup Language). The resulting package can run on an Android-powered device.

\section{RESULTS}

Figures 5 - 9 show screenshots of our Real-time tracking system for mobile phones.

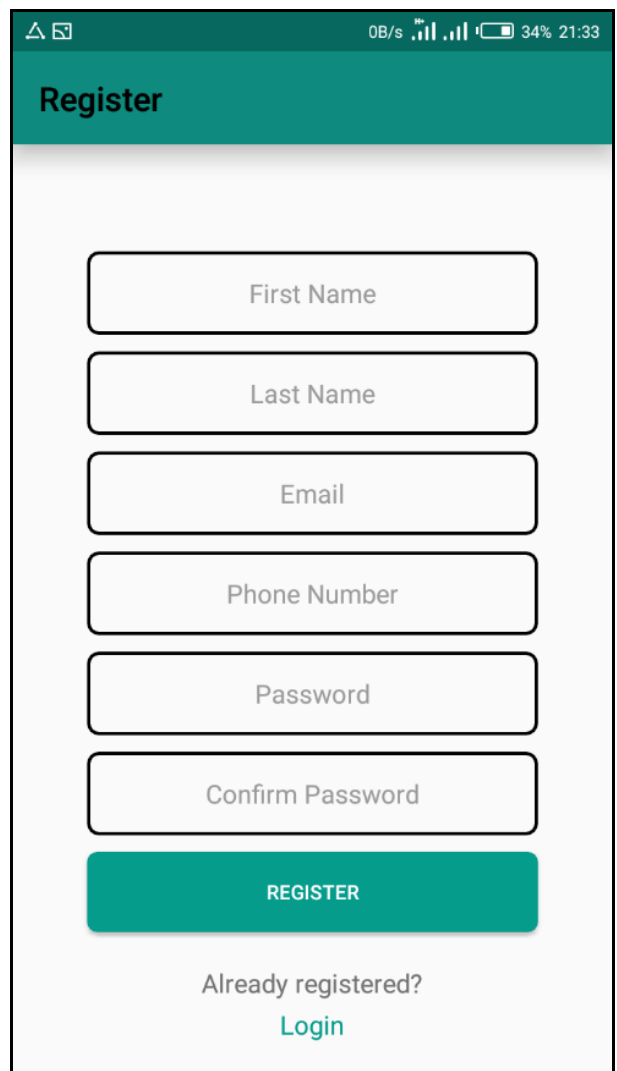

Figure 5: Registration interface screenshot

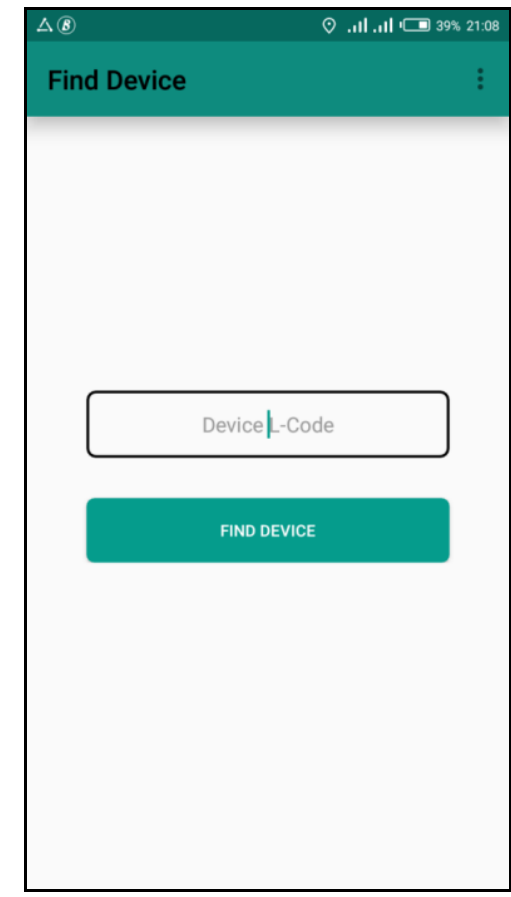

Figure 6: Find Device interface screenshot 


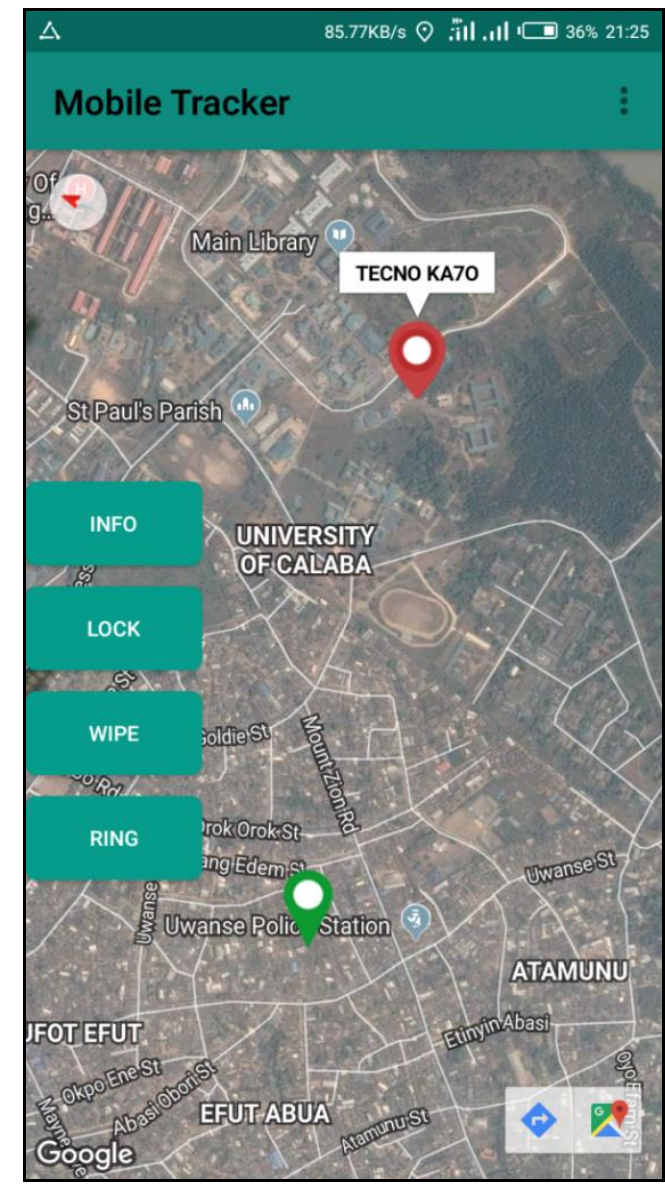

Figure 7: Control Device Interface screenshot

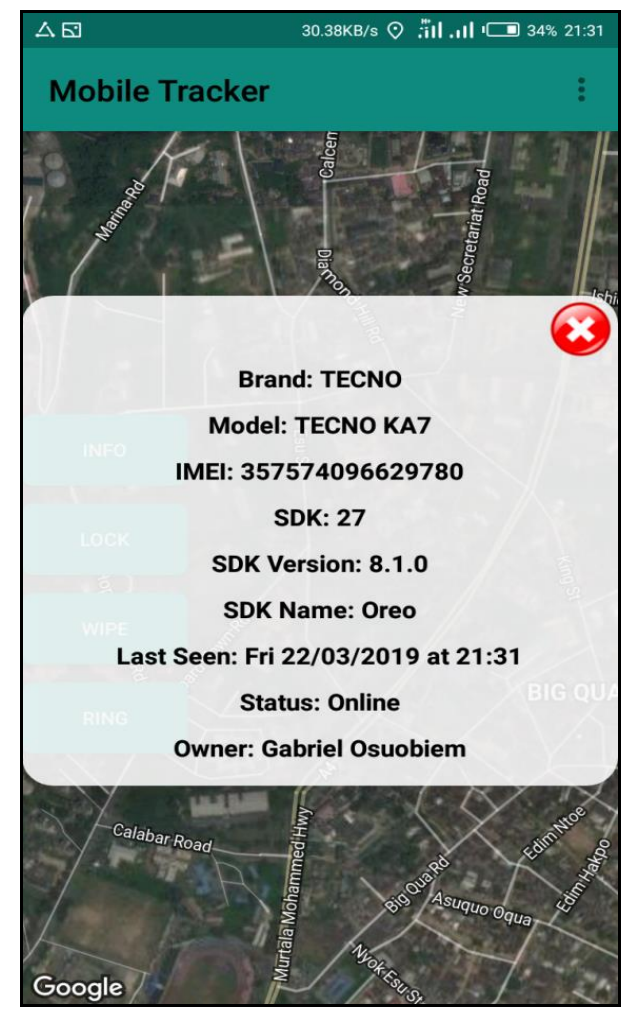

Figure 8: Device Details interface screenshot

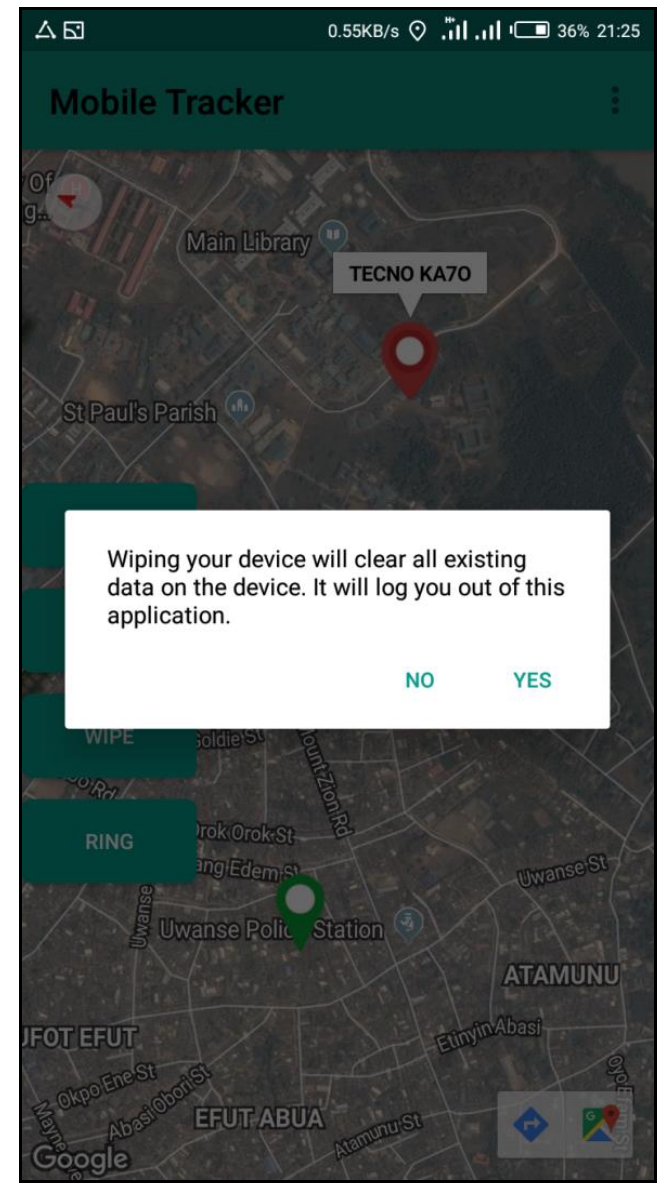

Figure 9: Confirm wipe interface screenshot

\section{DISCUSSION}

The application outputs shown in the Results section are explained next.

Registration Interface screenshot: This is where a user can register by filling the required fields in the form.

Find Device Interface screenshot: This interface allows a user to find a lost device by typing its L-CODE in the field provided. When the L-CODE is typed and has been verified, the application redirects the user back to the Map Interface.

Control Device Interface screenshot: This interface is invoked when a user has successfully found a device. It is part of the Map Interface. It has four buttons (INFO, LOCK, WIPE and RING), and it places a red marker on the map showing the current location of the lost device.

Device Details Interface screen shot: This interface shows details of the lost device. It pops up when the Info button on the Control Device Interface is clicked.

Confirm Wipe Interface screen shot: When a user clicks the WIPE button the Confirm Wipe Interface pops up showing a message of confirmation.

\section{CONCLUSION}

Recovery of misplaced or lost mobile phones is a rampant problem that requires address. This study was able solve the above-stated problem in a set of simplified steps. With the availability of the software developed in this study, finding a 
lost device has been simplified and less technical, so as to enable the most ignorant users recover their lost mobile phones.

\section{FUTURE RESEARCH}

Due to some technical and environmental limitations, several other features that are desired to be included in this research are lacking. Therefore, it is recommended that the following be included in future research:

- The map provided by the application when a device is found shows two markers (present device and lost device); it is desired that a line of direction is drawn from the source marker (device in use) to the destination marker (lost device) so as to give a user the knowledge of how to travel towards the lost device as its location changes. Future researchers should incorporate this feature to enhance user experience.

- When a device is to be registered by the system, the generated L-CODE is shown on the screen, this code consists of 20 characters which makes it difficult to remember. Future researchers should make a provision for the L-CODE to be sent to the user's email when a device is registered.

- A user can control a lost device only when there is internet connection; it is desired that, there be an SMS command feature. With this feature, a user should be able to control his/her device using text message commands. Future researchers should incorporate this feature as it will increase the chances of retrieving a lost device.

\section{REFERENCES}

[1] Smartphone. (2018). Retrieved October 22 2018, from Wikipedia: https://en.m.wikipedia.org/wiki/Smartphone

[2] Statista. (2018). Mobile OS market share 2018 | Statista. Retrieved from https://www.statista.com/statistics/266136/globalmarket-share-held-by smartphone-operating-systems/

[3] Deore, S., Khodade, K., \& Patil, S. (2017). Anti -Theft Application for Lost or Misplaced Android Phones. International Journal for Innovative Research in Multidisciplinary Field, 3(4).

[4] Raphael. (2017, August 17). Find My Device: How Android's security service can manage your missing phone Computerworld [Blog Post]. Retrieved from https://www.computerworld.com/article/3217025/find-my-deviceandroid-phone.html

[5] Google LLC. (2018). Find, lock, or erase your lost phone or computer - Google Account Help. Retrieved from https://support.google.com/accounts/answer/7177579?visit_id=636 911107053855687-395339011\&p=lda\&hl=en\&rd=

[6] Salim, S., Damodaran, D., \& Vargese, S. (2016). Mobile Tracker. International Journal on Cybernetics \& Informatics, 5(2).

[7] Statista. (2020). Smartphone users in Nigeria 2014-2025, Statista. Retrieved 11 March 2020 from https://www.statista.com/statistics/467187/forecast-of-smartphoneusers-in-nigeria/ 